\title{
Influencia de la invasión de Ligustrum lucidum (Oleaceae) sobre la comunidad de lianas en la sierra de San Javier (Tucumán - Argentina)
}

\author{
Sergio J. Ceballos ${ }^{\bowtie}$; Agustina Malizia \& Natacha P. Chacoff \\ CONICET. Instituto de Ecología Regional, Universidad Nacional de Tucumán, Argentina.
}

\begin{abstract}
Resumen. En la sierra de San Javier (Tucumán, Argentina) se presentan bosques secundarios nativos e invadidos, estos últimos dominados por Ligustrum lucidum, que afecta la biodiversidad nativa. Analizamos la influencia del bosque dominado por L. lucidum sobre las comunidades de lianas en comparación con bosques secundarios nativos sin dominancia de especies exóticas. Los bosques fueron comparados en abundancia, composición, modalidades de trepado y distribución de clases de tamaños de los tallos de lianas. Para ello se establecieron un total de 10 cuadrantes en el sector pedemontano de yungas de la sierra de San Javier en los cuales se midió, identificó y relevó los tallos de lianas $\geq$ a $0.5 \mathrm{~cm}$ y los árboles $\geq$ a $10 \mathrm{~cm}$ en diámetro a la altura del pecho. El bosque nativo presentó cuatro veces mayor abundancia y mayor riqueza de tallos de lianas en comparación con el bosque invadido. Los individuos de L. lucidum hospedaron tallos de lianas en muy baja abundancia y en el bosque dominado por esta especie exótica los hospedadores de lianas fueron principalmente árboles nativos. Además de no constituir soportes adecuados de lianas, se manifiesta una influencia indirecta a través de la exclusión de las especies de árboles nativos que las hospedan.
\end{abstract}

[Palabras clave: abundancia, árboles hospedadores, bosque invadido, bosques nativos, modalidades de trepado, riqueza, yungas]

\begin{abstract}
AвSTRACT. Influence of the invasion of Ligustrum lucidum (Oleaceae) on liana community in sierra de San Javier (Tucumán - Argentina). Native and invaded secondary forests are present in sierra de San Javier, the latter dominated by Ligustrum lucidum, which exerts an influence on native biodiversity. We analyzed the influence of the forest dominated by L. lucidum over liana communities compared to native secondary forests without dominance of exotic species. Forests were compared in abundance, composition, climbing mechanisms and distribution in size classes of liana stems. For this, liana stems $\geq 0.5 \mathrm{~cm}$ and the trees $\geq 10 \mathrm{~cm}$ in diameter at height breast, were measured, identified and surveyed in 10 plots located in the yungas piedmont of sierra de San Javier. Native forests had four times more abundance and richness of liana stems than invaded forest of $L$. lucidum. Individual trees of L. lucidum hosted liana stems in very low abundance, and in the forest dominated by this exotic species, the liana hosts were mainly native trees. Besides the fact that they do not constitute suitable hosts for lianas, they have an indirect influence through the exclusion of native host trees of lianas.
\end{abstract}

[Keywords: abundance, climbing mechanisms, host trees, invaded forest, native forests, richness, yungas]

\section{INTRODUCCIÓN}

Las lianas son plantas trepadoras leñosas que comparten una estrategia de crecimiento que consiste en ascender al dosel usando la arquitectura de otras plantas como soporte (Putz \& Holbrook 1991; Schnitzer \& Bongers 2002). Se consideran organismos intersticiales (Huston 1995) debido a que ocupan nichos generados por las especies estructurales y, como consecuencia, su abundancia responde a los cambios de composición y densidad de los árboles. Constituyen importantes elementos de los bosques tropicales y subtropicales, representando alrededor del $25 \%$ de la diversidad de especies leñosas, aunque este valor varía entre los distintos tipos de ambientes (Schnitzer \& Bongers 2002). Han sido objeto de estudio tanto en bosques tropicales y subtropicales (Putz 1984; PérezSalicrup \& de Meijere 2005; van der Heijden et al. 2008) como templados (Londré \& Schnitzer 2006).

Las investigaciones sobre las lianas han sido abordadas desde distintas perspectivas: sus interacciones con características de los árboles soporte y con la estructura de los bosques (Putz 1984; Clark \& Clark 1990; Malizia 2003; Malizia \& Grau 2006; Campanello et al. 2007), demografía (Putz 1990), ecología sucesional (Dewalt et al. 2000; Caballé \& Martin 2001), relaciones espaciales incluyendo claros naturales o antrópicos (Putz 1984; Malizia \& Grau 2008; Ding \& Zang 2009), y su relación con el contenido de nutrientes del suelo (Malizia et al. 2010), entre otras. La mayoría de los estudios sobre las comunidades de lianas se hicieron en bosques nativos (Putz 1984; Caballé \& Martin 2001; Pérez-Salicrup \& de Meijere 2005; van der Heijden et al. 2008) 
y en bosques disturbados, por ejemplo por la tala selectiva o clareo (Campanello et al. 2007; Ding \& Zang 2009), sin considerar otro tipo de impactos sobre las comunidades de estas plantas trepadoras.

Un impacto importante sobre las comunidades de plantas nativas es la invasión biológica de especies exóticas, que trae como consecuencia la modificación del ecosistema en general. La invasión de especies de árboles exóticos en los bosques tiene la tendencia a afectar la estructura comunitaria de las lianas directa o indirectamente y sus relaciones con sus hospedadores (Addo-Fordjour et al. 2009). En bosques de Ghana, Broussonetia papyrifera (L.) Vent. (Moraceae) es una planta exótica invasora que afecta a las comunidades de lianas, debido a que limita el número de soportes disponibles en los bosques y aparentemente no es una hospedadora adecuada (Addo-Fordjour et al. 2009). Sin embargo, se ha señalado que poco se conoce sobre el efecto de las invasiones biológicas sobre estas plantas trepadoras (Addo-Fordjour et al. 2009).

Ligustrum lucidum W.T. Aiton es un especie leñosa, originaria del Sudeste Asiático, que invadió exitosamente bosques chaqueños serranos, yungas y un relicto de selva ribereña (Punta Lara) en Argentina (Grau \& Aragón 2000; Montaldo 2000; Lichstein et al. 2004; Giorgis et al. 2011a; Gavier-Pizarro et al. 2012). En bosques chaqueños serranos de las sierras de Córdoba, L. lucidum se estableció en comunidades naturales y suburbanas (Giorgis et al. 2011b; Gavier-Pizarro et al. 2012; Furey et al. 2014) formando parches densos monoespecíficos (Giorgis et al. 2011a; Gavier-Pizarro et al. 2012), disminuyendo la cobertura de los estratos herbáceo y arbustivo, y modificando los patrones de estructura, diversidad y regeneración de los bosques nativos (Hoyos et al. 2010). En el relicto de selva ribereña de Punta Lara (Buenos Aires), la invasión de L. lucidum se vio favorecida por la marginalidad ecológica de la comunidad selvática, ya quemuchas especiesseencuentran en el extremo meridional de su distribución (Montaldo 2000). En las yungas de la sierra de San Javier (Tucumán, Argentina), L. lucidum invadió bosques sucesionales jóvenes y áreas abandonadas por la agricultura y ganadería, ocasionando cambios en el hábitat (e.g. simplificación estructural) afectando a la flora y la fauna nativas (e.g. aves), el ciclo del agua, la descomposición de hojas, entre otras (Grau \& Aragón 2000; Lichstein et al. 2004; Aragón et al. 2014; Ayup et al. 2014; Zamora Nasca et al. 2014), dando lugar a "nuevos bosques secundarios" diferentes a los nativos.

En las yungas de la sierra de San Javier, los patrones de reclutamiento de las especies leñosas se han visto modificados y a medida que la especie invasora incrementa su dominancia, la abundancia de lianas disminuye (Lichstein et al. 2004). Se ha señalado que las características de L. lucidum como la corteza lisa y sus ramas a gran altura pueden llegar a dificultar la expansión de las lianas en estos bosques invadidos (Lichstein et al. 2004), sin embargo hasta el momento no se han realizados estudios detallados que evalúen el efecto de L. lucidum sobre la comunidad de lianas. El objetivo general de este trabajo es evaluar la influencia que el bosque invadido por L. lucidum ejerce sobre las comunidades de lianas en comparación con bosques secundarios nativos.

Los objetivos específicos buscaron: (1) Comparar la abundancia y riqueza de lianas en bosques nativos y en un bosque invadido por L. lucidum del pedemonte de la sierra de San Javier; (2) Analizar las diferencias en las modalidades de trepado y distribución de lianas en clases de tamaño entre los dos tipos de bosque; y (3) Examinar la relación entre el tamaño de las lianas y características físicas de los árboles hospederos (diámetro, altura total, altura hasta la primera ramificación y tasa de crecimiento) tanto en bosques nativos como en el bosque invadido.

La hipótesis general es que L. lucidum afecta a las comunidades de lianas a través de su influencia a nivel individual (e.g. ofreciendo pocas oportunidades para el reclutamiento al no constituir hospedadores adecuados) y de comunidad (e.g. excluyendo a las especies nativas hospedadoras de lianas). Como consecuencia se predice que en los bosques invadidos por L. lucidum: (1) se observará una disminución en la abundancia y en la riqueza de lianas en comparación con los bosques nativos. (2) Las lianas tendrán diámetros pequeños debido a la dificultad de alcanzar un gran crecimiento en los bosques invadidos y con modalidades predominantemente adecuadas al trepado de soportes de menor diámetro (e.g. zarcillos). (3) L. lucidum presenta características que dificultan el trepado de lianas (e.g. rápido crecimiento y pocas ramas a baja altura) y que tendrán una influencia en la disminución de la abundancia de estas plantas trepadoras en los bosques invadidos. 


\section{MÉTODOS}

\section{Área de estudio}

Se trabajó en la zona pedemontana de la ladera Este de la sierra de San Javier en la provincia de Tucumán $\left(26^{\circ} 47^{\prime} \mathrm{S}, 65^{\circ} 22^{\prime} \mathrm{O}\right)$, donde se localiza el Parque Biológico homónimo, área protegida que pertenece a la Universidad Nacional de Tucumán (Brown et al. 2002). El clima es estacional, con un verano cálido y húmedo y un invierno fresco y seco, la temperatura media anual ronda $\operatorname{los} 18^{\circ} \mathrm{C}$ y las precipitaciones ocurren bajo un régimen monzónico con alrededor de $1400 \mathrm{~mm}$ anuales (Bianchi 1981). La zona forma parte de la provincia fitogeográfica de las yungas, una región selvática caracterizada por un dosel arbóreo dominado por especies nativas que alcanzan gran porte, como Cinnamomum porphyrium (Griseb.) Kosterm (Lauraceae) y Blepharocalyx salicifolius (Kunth) O. Berg (Myrtaceae) y varios estratos de árboles, arbustos y renovales en niveles inferiores, con presencia de lianas y epífitas (Cabrera 1971; Grau et al. 2010). Un gran porcentaje del área fue deforestada durante la primera mitad del siglo XX para cultivos de herbáceas y frutales, principalmente cítricos, y luego con la pérdida de productividad del suelo experimentó una revegetación natural (Grau et al. 1997). Los bosques secundarios ocuparon estas áreas abandonadas, algunos de ellos dominados por especies exóticas, principalmente Ligustrum lucidum, resultando en un mosaico de bosques secundarios con y sin dominio de especies exóticas (Grau et al. 2008).

\section{Recopilación de datos}

Se relevaron 10 cuadrantes dentro de bosques secundarios entre 25 y 30 años de edad en el sector pedemontano de las yungas de la sierra de San Javier: 5 de bosques secundarios nativos y 5 de un bosque invadido por L. lucidum. Estos bosques se encuentran en similares condiciones de pendiente $(10 \%)$ y altitud $(720 \mathrm{~m})$ y sus coordenadas son: $26^{\circ} 46^{\prime} 58.2^{\prime \prime} \mathrm{S} 65^{\circ} 19^{\prime} 46.4^{\prime \prime} \mathrm{O}$ y $26^{\circ} 46^{\prime} 08.3^{\prime \prime} \mathrm{S}$ $65^{\circ} 19^{\prime} 22.9^{\prime \prime} \mathrm{O}$ (bosques nativos) y $26^{\circ} 46^{\prime} 23.5^{\prime \prime} \mathrm{S}$ 6519'22.1" O (bosque invadido).

Todos los cuadrantes tuvieron una dimensión de $20 \times 20 \mathrm{~m}$ y estuvieron separados por una distancia mínima de $40 \mathrm{~m}$ para evitar muestrear dos veces el mismo individuo. Dentro de cada cuadrante, se relevaron todos los tallos de lianas $\geq$ a $0.5 \mathrm{~cm}$ de diámetro libres y los asociados a los árboles, identificando la especie y midiendo su diámetro con un calibre a $20 \mathrm{~cm}$ a partir del punto de enraizamiento más evidente (i.e. donde no se vieron más conexiones). Para el inventario de lianas se siguió el protocolo de Gerwing y colaboradores (2006), sin considerar su recomendación sobre la ubicación del punto de medición (i.e. no hubo sobreestimación de la densidad de lianas midiéndolas a $20 \mathrm{~cm}$ - datos no publicados). Se prefirió trabajar con tallos de lianas debido a la dificultad de individualizar las lianas en el campo. La información sobre su modalidad de trepado (espinas, ramas divaricadas, uncinulos y zarcillos) fue obtenida en el campo y de la bibliografía (Ayarde 2005). Los árboles $\geq$ a $10 \mathrm{~cm}$ de diámetro a la altura del pecho (dap), con o sin lianas, fueron identificados a nivel de especie, se midió su diámetro, se estimó su altura total y la altura hasta la primera ramificación, excepto la información sobre el dap de los árboles del bosque invadido que fueron obtenidos de la base de datos de parcelas permanentes que posee el Instituto de Ecología Regional (UNT). También, se recopilaron datos de la tasa de crecimiento máximo en bosques secundarios de las especies de árboles (Easdale et al. 2007). Finalmente, en cada cuadrante se realizaron 2 transectas para medir la cobertura del sotobosque bajo ( $<2 \mathrm{~m}$ de altura) y alto ( $>2 \mathrm{y}<6 \mathrm{~m}$ de altura). Cada transecta tuvo una longitud de $20 \mathrm{~m}$ y con la ayuda de una vara se registraron los toques de la vegetación del sotobosque alto y bajo en 10 puntos distanciados cada $2 \mathrm{~m}$. Los resultados de ambas transectas se promediaron para obtener los porcentajes de cobertura del sotobosque alto y bajo para cada cuadrante.

\section{Análisis de datos}

Para comparar los bosques nativos con el bosque invadido en relación a la abundancia y composición florística de tallos de lianas (objetivo 1), se utilizaron dos tipos de estadísticos diferentes. La comparación de la abundancia de tallos de lianas se realizó a través de un Test U de Mann-Whitney, mientras que los cambios en composición específica entre los bosques se analizaron ordenando los cuadrantes relevados $(n=10)$ por medio de un NMDS (NonMetrical Multidimensional Scaling) basado en una matriz de similitud de Bray Curtis entre los cuadrantes calculada a partir de la abundancia de especies de lianas. Para mejorar la convergencia del NMDS, antes de correr el análisis se eliminó una especies presente en un solo cuadrante (Pisoniella arborescens), y las abundancias fueron transformadas a logaritmo $(X+1)$ para reducir la variabilidad dentro y entre especies. Se utilizó el modo autopiloto (modo "lento y completo") para decidir cual dimensión era la mejor solución (McCune \& Grace 2002). La elección de dos dimensiones se debió a que el stress final (un índice de la concordancia entre las distancias en el espacio del ordenamiento y las distancias en la matriz de Bray Curtis) fue 8.65 ( $<20$ es considerado aceptable para set de datos ecológicos) y fue diferente del azar (test de Monte Carlo = 250 corridas con la matriz randomizada $\mathrm{p}=0.04)(\mathrm{McC}$ une \& Grace 2002). El NMDS considera similares a los cuadrantes con una abundancia de especies de lianas comparable, y como resultado se presentan cercanos en el espacio del ordenamiento. Para determinar cuáles especies de lianas influyeron en el ordenamiento, se calculó su posición sobre cada eje con el método de "pesado promedio" en donde los valores de los cuadrantes sobre los ejes se usan como pesos para encontrar la posición promedio de cada especie, la cual está representada por curvas de abundancia unimodales no lineales a lo largo de los ejes 
(McCune \& Grace 2002). Finalmente, se calculó el coeficiente de determinación (porcentaje de varianza representada por cada eje) entre las distancias originales en la matriz de Bray Curtis y las distancias en el espacio del ordenamiento de cada cuadrante sobre cada uno de los ejes (McCune \& Grace 2002).

Las diferencias entre los bosques nativos y el bosque invadido en relación a las proporciones de las modalidades de trepado y en la distribución de tallos de lianas en clases de tamaño (objetivo 2) se analizaron a través de un Chi Cuadrado. De este modo se evaluó si las modalidades de trepado o clases de tamaños de estas plantas son independientes del tipo de bosque (nativo o invadido). Las clases de tamaño consideradas fueron: 0.5 a $2 \mathrm{~cm},>2$ a $4 \mathrm{~cm},>4$ a $6 \mathrm{~cm}$ y $>6 \mathrm{~cm}$.

Para examinar la relación entre el tamaño de los tallos de lianas (diámetro) y los determinantes físicos considerados para el trepado de los mismos se efectuaron una serie de regresiones múltiples para ambos tipos de bosque (objetivo 3). Como determinantes físicos (variables explicativas) se consideraron características de los árboles soporte: diámetro a la altura del pecho, altura total, altura del fuste hasta la primera ramificación y crecimiento máximo. Previo a los análisis las variables fueron estandarizadas con respecto a la media (Gelman \& Hill 2007). Luego de realizadas las regresiones se utilizó la función step disponible en el paquete stat (R Core Team 2013) para obtener el mejor modelo explicativo.

Finalmente los bosques nativos y el bosque invadido fueron comparados en su densidad de tallos, densidad de individuos, riqueza, altura total, altura hasta la primera ramificación, dap promedio, cobertura del sotobosque alto y cobertura del sotobosque bajo por medio del estadístico U de Mann-Whitney.
Todos los análisis de datos, con excepción del ordenamiento, se efectuaron con el programa estadístico R (R Core Team 2013). El programa estadístico empleado para el ordenamiento fue PC-ORD 5.0 (McCunne \& Mefford 2006).

\section{Resultados}

Los bosques nativos relevados tuvieron una riqueza de 16 especies de árboles entre los cuales se destacaron por su dominancia Parapiptadenia excelsa (Griseb.) Burkart (Fabaceae) y Cinnamomum porphyrium (Lauraceae) en el dosel y Piper tucumanum C. DC. (Piperaceae) y Allophyllus edulis (A. St.Hil., A. Juss \& Cambess.) Hieron. ex Niederl (Sapindaceae) en el estrato arbustivo. Estos bosques alcanzaron una altura promedio de $9 \mathrm{~m}$ y una densidad media de 15 individuos y 17 tallos por cuadrante. El bosque invadido relevado presentó una gran dominancia de Ligustrum lucidum (más del 50\% del total de individuos), mientras que la especie nativa más común fue Myrsine laetevirens (Mez) Arechav. (Myrsinaceae). El bosque invadido tuvo menor riqueza (8 especies de árboles), mayor altura, densidad de individuos y número de tallos de árboles por cuadrante (Tabla 1).

Los bosques nativos y el bosque invadido del área de estudio difirieron en su abundancia de tallos de lianas $(\mathrm{W}=24.5 \mathrm{p}$ $=0.016)$. La abundancia de tallos de lianas en los bosques nativos fue 4 veces mayor que la del bosque invadido (Figura 1). Las especies de lianas registradas en el bosque invadido fueron seis: Dolichandra unguis-cati (L.) L.G. Lohmann (Bignoniaceae), Vernonia

Tabla 1. Comparaciones entre los atributos del bosque nativo y el bosque invadido por medio del Test de MannWhitney. Las variables incluidas hacen referencia únicamente a los árboles. Las diferencias significativas ( $\mathrm{p} \leq 0.05$ ) están señaladas con asterisco.

Table 1. Comparisons between the attributes of the native and invaded forests with Mann-Whitney Test. The variables included refer only to trees. Significant differences $(p \leq 0.05)$ are indicated with an asterisk.

\begin{tabular}{|c|c|c|c|}
\hline & Bosque Nativo & Bosque Invadido & Test de Mann-Whitney \\
\hline Riqueza total & 16 & 8 & \\
\hline Riqueza promedio por cuadrante & $6.8 \pm 0.86$ & $4.8 \pm 0.58$ & $\mathrm{~W}=22 \mathrm{p}=0.04^{*}$ \\
\hline $\begin{array}{l}\text { Densidad de individuos por } \\
\text { cuadrante }\end{array}$ & $15 \pm 1.97$ & $21.8 \pm 2.85$ & $W=5 p=0.14$ \\
\hline Densidad de tallos por cuadrante & $17 \pm 2.14$ & $24.2 \pm 3.15$ & $\mathrm{~W}=4 \mathrm{p}=0.08$ \\
\hline Promedio de dap $(\mathrm{cm})$ & $21.5 \pm 1.44$ & $25 \pm 1.02$ & $W=8 p=0.42$ \\
\hline Promedio de altura total (m) & $8.8 \pm 0.38$ & $11.6 \pm 0.36$ & $\mathrm{~W}=25 \mathrm{p}=0.01^{*}$ \\
\hline $\begin{array}{l}\text { Promedio de altura hasta la primera } \\
\text { ramificación }(\mathrm{m})\end{array}$ & $3.7 \pm 0.26$ & $5.7 \pm 0.26$ & $\mathrm{~W}=0 \mathrm{p}=0.01^{*}$ \\
\hline Cobertura del sotobosque alto & $62 \pm 9.17$ & $24 \pm 9.27$ & $\mathrm{~W}=23 \mathrm{p}=0.03^{*}$ \\
\hline Cobertura del sotobosque bajo & $76 \pm 6.78$ & $44 \pm 6.78$ & $\mathrm{~W}=24 \mathrm{p}=0.02^{*}$ \\
\hline Especies dominantes & $\begin{array}{c}\text { Piper tucumanum, } \\
\text { Parapiptadenia excelsa y } \\
\text { Cinnamomum porphyrium. }\end{array}$ & $\begin{array}{l}\text { Ligustrum lucidum, Myrsine } \\
\text { laetevirens y Morus alba. }\end{array}$ & \\
\hline
\end{tabular}




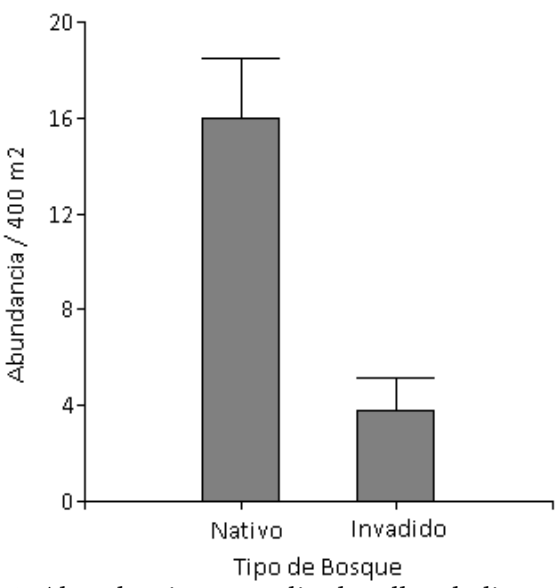

Figura 1. Abundancia promedio de tallos de lianas por tipo de bosque. Las barras representan los bosques nativo e invadido con su correspondiente error estándar.

Figure 1. Average abundance of liana stems by forest type. The bars represent native and invaded forests with their standar error.

fulta Griseb. (Asteraceae), Rubus imperialis Chad. \& Schltdl (Rosaceae), Smilax campestris Griseb. (Smilacaceae), Cissus striata Ruiz \& Pav. (Vitaceae) y Celtis iguanaea (Jacq.) Sarg (Celtidaceae). Las especies de lianas registradas en los bosques nativos fueron diez: D. unguis-cati, Chamissoa altissima (Jacq.) Kunth (Amaranthaceae), V. fulta, C. striata, Hebanthe occidentalis (R.E. Fr.) Borsch \& Pedersen (Amaranthaceae), Serjania meridionalis Cambess. (Sapindaceae), R. imperialis, $S$. campestris, C. iguanaea y Pisoniella arborescens (Lag. \& Rodr.) Standl. (Nyctaginaceae) (Tabla 2). Los cuadrantes de los diferentes bosques tendieron a segregarse a lo largo de dos ejes del NMDS basado en la composición especifica de lianas (varianza representada por el eje $1=$ $57 \%$ y varianza representada por el eje $2=35 \%$, varianza total representada por ambos ejes $=$ $92 \%$.) (Figura 2). En el gráfico, los cuadrantes de bosques nativos estuvieron agrupados principalmente en los sectores positivos del eje 1 y el eje 2, mientras que los cuadrantes del bosque invadido se distribuyeron de un modo disperso a lo largo de ambos ejes. Dolichandra unguis-cati, V. fulta, H. occidentalis, S. meridionalis y C. altissima presentaron mayor abundancia hacia los cuadrantes ubicados en los sectores positivos de los ejes 1 y 2 , es decir, donde se encuentran los cuadrantes de bosques nativos. Smilax campetris y $R$. imperialis tuvieron mayor abundancia en el sector negativo del eje 1 , donde se disponen los cuadrantes del bosque invadido Lig 3 y Lig 5. Los cuadrantes con menor riqueza y abundancia de lianas (Lig 1, Lig 4 y Lig 5) se dispusieron en los extremos negativos de ambos ejes.

Las modalidades de trepado de los tallos de lianas difirieron entre los bosques nativos y el bosque invadido $\left(X^{2}=19.83 p<0.01\right)$. En ambos bosques predominó la presencia de uncinulos debido a la abundancia de Dolichandra unguiscati. En los bosques nativos fueron más comunes las ramas divaricadas (i.e. apoyantes) y en el bosque invadido se presentaron en mayor proporción las modalidades con espinas y zarcillos (Figura 3). La mayoría de los tallos de lianas estuvieron trepados a los árboles (66\% en bosques nativos y $72 \%$ en el bosque invadido), presentándose los tallos libres en menor proporción. En general las lianas de tallos libres presentaron un diámetro

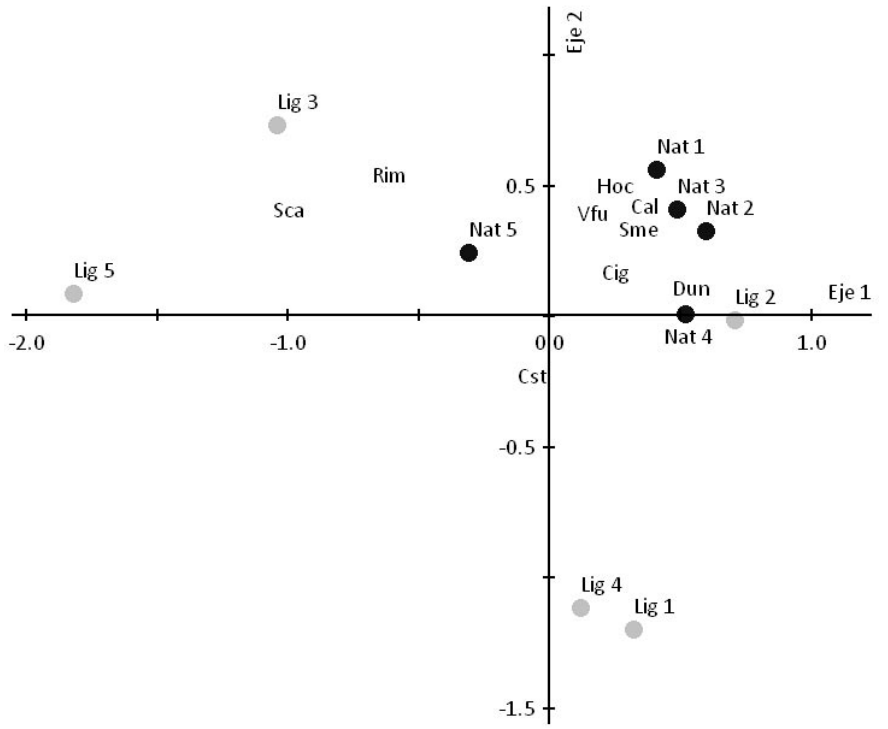

Figura 2. Diagrama de ordenamiento (NMDS) de los cuadrantes de los bosques nativo e invadido y las especies de lianas. Referencias: Cal (Chamissoa altissima), Cig (Celtis iguanaea), Cst (Cissus striata), Dun (Dolichandra unguis-cati), Hoc (Hebanthe occidentalis), Par (Pisoniella arborescens), Rim (Rubus imperialis), Sca (Smilax campestris), Sme (Serjania meridionalis) Vfu (Vernonia fulta).

Figure 2. Ordinatio diagram (NMDS) of native and invaded forest plots and liana species. References as in Spanish legend. 
Tabla 2. Especies de lianas registradas en los bosques nativos y en el bosque invadido, listadas por familia y mecanismo de trepado. La información fue obtenida de Ayarde 2005.

able 2. Liana species registered in native and invaded forests, listed by family and climbing mechanism. The data was obtained from Ayarde 2005.

\begin{tabular}{lll}
\hline Especie & Familia & Mecanismo de trepado \\
\hline Celtis iguanaea (Jacq.) Sarg & Celtidaceae & Espinas \\
Chamissoa altissima (Jacq.) Kunth & Amaranthaceae & Ramas divaricadas \\
Cissus striata Ruiz \& Pav. & Vitaceae & Zarcillos \\
Dolichandra unguis-cati (L.) L.G. Lohmann & Bignoniaceae & Uncínulos \\
Hebanthe occidentalis (R.E. Fr.) Borsch \& Pedersen & Amaranthaceae & Ramas divaricadas \\
Pisoniella arborescens (Lag. \& Rodr.) Standl. & Nyctaginaceae & Ramas divaricadas \\
Rubus imperialis Chad. \& Schltdl & Rosaceae & Espinas \\
Serjania meridionalis Cambess. & Sapindaceae & Zarcillos \\
Smilax campestris Griseb. & Smilacaceae & Zarcillos \\
Vernonia fulta Griseb. & Asteraceae & Ramas divaricadas \\
\hline
\end{tabular}

pequeño (e.g. $x=1,6 \mathrm{~cm}$ ), pudiendo tratarse de individuos que todavía no alcanzaron soportes o bien, brotes de lianas de mayor diámetro. En el bosque invadido, de un total de 220 individuos de Ligustrum lucidum relevados, solo dos de ellos $(0.9 \%)$ fueron hospedadores de tallos de lianas. En este bosque, las especies de árboles hospedadoras de tallos de lianas fueron principalmente especies nativas como Blepharocalyx salicifolius, Myrsine laetevirens, Cinnamonum porphyrium, Parapiptadenia excelsa y Cupania vernalis Cambess. y la exótica Morus alba L. (mora). El 21\% de los individuos nativos y el 13\% de las moras relevadas fueron hospedadores de tallos de

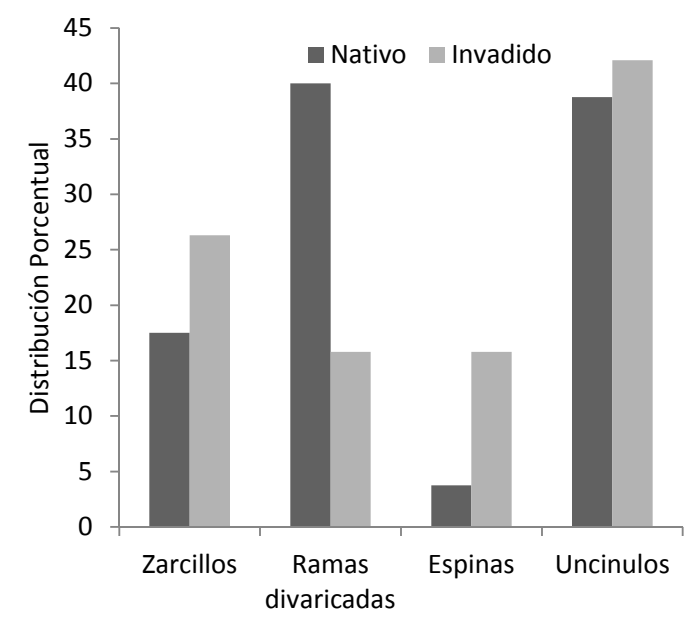

Figura 3. Distribución porcentual de las modalidades de trepado de los tallos de lianas por tipo de bosque en la sierra de San Javier, Tucumán. Las barras gris oscuro representan al bosque nativo y las barras gris claro representan al bosque invadido.

Figure 3. Percentage distribution of the climbing mechanisms of liana stems by forest type in sierra de San Javier, Tucumán. The dark gray bars represent the native forest and the light gray bars represent the invaded forest.

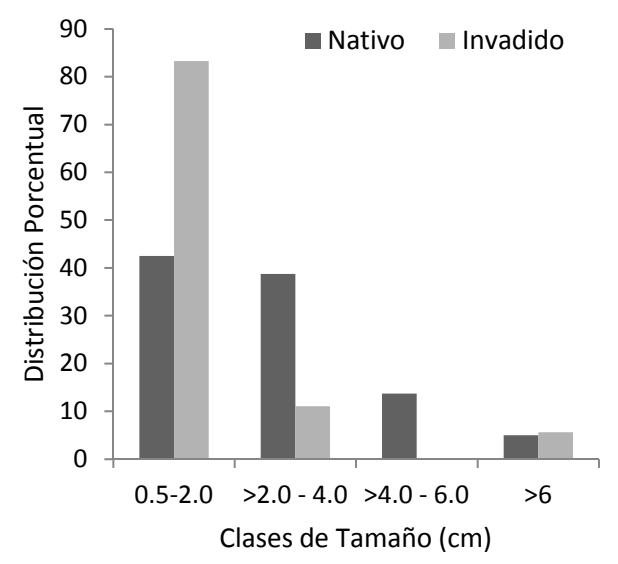

Figura 4. Distribución de clases de tamaño de los tallos de lianas por tipo de bosque en la sierra de San Javier, Tucumán. Las barras gris oscuro representan al bosque nativo y las barras gris claro representan al bosque invadido.

Figure 4. Size class distribution of liana stems by forest type in sierra de San Javier, Tucumán. The dark gray bars represent the native forest and the light gray bars represent the invaded forest.

lianas en el bosque invadido. Las especies de árboles más abundantes en los bosques nativos, P. excelsa y C. porphyrium en el dosel y Piper tucumanum en el sotobosque, hospedaron una mayor cantidad de lianas ( $42 \%$ del total). Cabe destacar que en los bosques nativos se relevaron especies exóticas hospedadoras como M. alba y Persea americana Mill.

La distribución de los tallos de lianas en clases de tamaño difirió entre los bosques $\left(\mathrm{X}^{2}\right.$ $=42.35 \mathrm{p}<0.01)$. En el bosque invadido, el $80 \%$ de los tallos de lianas tuvieron un diámetro entre 0.5 y $2 \mathrm{~cm}$. En cambio, en los bosques nativos la curva de las clases de tamaño fue más suavizada, encontrándose la mayoría de las lianas entre las clases de 0.5 a $4 \mathrm{~cm}$ de diámetro (Figura 4). 
En los bosques nativos, el diámetro o tamaño de los tallos de lianas estuvo explicado en un $32 \%$ por el diámetro de los hospedadores $(\beta=$ $0.066 \pm 0.014)$ y con su altura hasta la primera ramificación $(\beta=0.16 \pm 0.09)$. En cambio, en el bosque invadido el diámetro de los tallos de lianas no se relacionó con ninguna de las características consideradas de los árboles hospedadores. El resto de las variables consideradas (tasa de crecimiento y altura total de los árboles), fueron eliminadas por la función step debido a su baja contribución en la explicación del diámetro de los tallos de lianas.

Los bosques nativos y el bosque invadido presentaron diferente riqueza, altura total del dosel, altura hasta la primera ramificación de los árboles, cobertura del sotobosque alto y cobertura del sotobosque bajo, mientras que no se diferenciaron en la densidad de tallos, densidad de individuos y en el dap promedio de los árboles (Test de Mann-Whitney, Tabla 1). De este modo, se puede inferir que la mayor abundancia de tallos de lianas estuvo relacionada con un bosque más diverso, de menor altura, con ramificaciones más bajas y un sotobosque más denso. Estas características estuvieron más representadas en los bosques nativos que en el invadido.

\section{DisCUSIÓN}

En el pedemonte de la sierra de San Javier se presentan bosques secundarios nativos e invadidos, estos últimos dominados por Ligustrum lucidum. Esta especie exótica invasora afecta el reclutamiento de las especies leñosas nativas y tiene un efecto sobre las lianas, cuya abundancia decrece con la dominancia (e.g. área basal) de $L$. lucidum (Lichstein et al. 2004). Se ha señalado que su dominancia es un buen predictor de la abundancia de las lianas probablemente debido a sus características: corteza lisa y ramas bajas escasas que dificultan el trepado de las lianas hacia el dosel (Lichstein et al. 2004). Observamos que el bosque invadido posee una baja abundancia de tallos de lianas en comparación con los bosques nativos y que en menos del 1\% de los individuos de L. lucidum relevados trepaban tallos de lianas. Resultados similares se obtuvieron en una investigación en bosques de Ghana, en la cual se observó que la planta invasora Broussonetia papyrifera (Moraceae) no sostuvo ninguna liana, indicando que la especie no es una hospedadora adecuada (Addo-Fordjour et al. 2009).
Ligustrum lucidum cuenta con una serie de ventajas para su expansión y el mantenimiento de sus poblaciones, como una eficiente dispersión por semillas, o rebrotes vegetativos, rápida tasa de crecimiento y tolerancia a la sombra. Puede sobrevivir en un amplio rango de hábitats y probablemente suprima la regeneración de las especies nativas por competencia (Grau \& Aragón 2000; Aragón \& Groom 2003; Easdale et al. 2007; Ayup et al. 2014). Por estos motivos, y en particular por su capacidad de auto-remplazo (Grau \& Aragón 2000), es probable que su influencia sobre las comunidades de lianas se mantenga en el tiempo. La dominancia de L. lucidum se refleja en la escasa proporción de árboles nativos relevados en el bosque invadido (17\%). Blepharocalyx salicifolius, Myrsine laetevirens, Cinnamonum porphyrium, Parapiptadenia excelsa y Cupania vernalis, fueron las especies de árboles nativos relevadas en el bosque invadido y prácticamente los únicos hospederos de lianas en este bosque.

El número de potenciales hospedadores disponibles afecta la abundancia de lianas (Campanello et al. 2007; Addo-Fordjour et al. 2009), de modo que mientras un bosque presente mayor número de árboles, mayor será la abundancia de lianas. Comparando los bosques nativos con el bosque invadido, se observó que esta relación no fue necesariamente de esta manera dado que una hectárea de bosque nativo tiene unos 375 árboles y el bosque invadido tiene alrededor de 562 individuos.

La estructura de los bosques y su luminosidad influencian la abundancia de lianas (Putz 1984; Gentry 1991; DeWalt et al. 2000). En el caso de los bosques invadidos por L. lucidum, la luminosidad que llega al sotobosque es baja, lo cual disminuye la cobertura de este estrato y favorece la dominancia de la especie nativa Psychotria carthagenensis Jacq. (Ayup et al. 2014), que podría constituir un soporte de baja altura disponible para ser trepado por lianas (Malizia \& Grau 2008). Se detectó que los tallos de lianas en los bosques estudiados parecen responder a la cobertura del sotobosque bajo debido a que utilizan este estrato para trepar y alcanzar árboles de mayor altura (Putz 1984; Ayarde 2005). Su abundancia se incrementa en los claros de los bosques (Putz 1984; Malizia \& Grau 2008; Malizia et al. 2010) y su crecimiento se reduce en áreas sombreadas (Putz 1990). Debido a que son marcadamente heliófitas, su abundancia puede reflejar el grado de cobertura del dosel (Caballé \& Martin 2001; 
Londré \& Schnitzer 2006; Malizia \& Grau 2006). Además, los bosques invadidos por $L$. lucidum son más oscuros, debido a su dosel cerrado (Grau \& Aragón 2000; Lichstein et al. 2004; Ayup et al. 2014) y a que presentan menos claros (Ceballos obs. pers.). Los claros, que representarían la oportunidad de las lianas para el ingreso en el bosque invadido, son rápidamente cubiertos debido que L. lucidum puede auto-reemplazarse y crecer rápidamente alcanzando una gran altura, disminuyendo la luminosidad de los bosques que requieren las lianas para su establecimiento, crecimiento y proliferación (Ceballos obs. pers.). Por ello se plantea que las lianas del bosque invadido en el área de estudio, probablemente ingresaron en condiciones de menor luminosidad o asociadas a claros que fueron cubiertos rápidamente.

Los tallos de lianas alcanzaron mayor diámetro cuando se encontraron asociados a árboles más grandes en diámetro. Las investigaciones encontraron que la infestación por lianas y su área basal se incrementan con el diámetro de sus hospedadores (Clark \& Clark 1990; Campanello et al. 2007; van der Heijden et al. 2008, Malizia \& Grau 2006). Los árboles de mayor diámetro usualmente cargan con más individuos y mayor área basal de lianas porque tienen más probabilidad de estar expuestos a la luz y experimentaron mayor tiempo de exposición a estas plantas trepadoras (Pérez-Salicrup \& de Meijere 2005; Malizia \& Grau 2006). En este trabajo se observó esta relación solo en el caso de los bosques nativos. En el bosque invadido la relación entre el diámetro de los tallos de lianas y el tamaño de los árboles probablemente no pudo detectarse debido al bajo número de individuos hospedadores de lianas en este tipo de bosque. Si bien se encontraron lianas asociadas a los árboles de mayor tamaño, otras investigaciones observaron que estas plantas proliferan mejor bajo doseles más bajos, con mayor disponibilidad de luz (Hegarty \& Caballé 1991; Malizia et al. 2009).

Caballé \& Martin (2001) consideran que las lianas que no tienen órganos especializados tienen mayor potencial para adaptarse y habitar distintos tipos de bosques. Sin embargo, aquí observamos que las lianas que predominaron en los dos ambientes fueron aquellas que cuentan con órganos especializados (uncinulos, zarcillos, espinas). Dolichandra unguis-cati fue la especie más abundante y debido a que cuenta con uncinulos, esta modalidad de trepado fue la más difundida en ambos tipos de bosque. Con respecto a las otras modalidades de trepado, las lianas con zarcillos predominaron más en el bosque invadido que en los bosques nativos, probablemente debido a que requieren de soportes de menor diámetro para trepar (Putz 1984; DeWalt et al. 2000). Además de las lianas con uncinulos, en los bosques nativos fueron frecuentes también las lianas con ramificaciones (i.e. apoyantes), que requieren distintos tipos de soporte, algunos de ellos de un tamaño importante para poder sostener sus tallos. Las formas libres tuvieron menor proporción en ambos tipos de bosque y presentaron diámetros pequeños, pudiendo tratarse de lianas que todavía no alcanzaron soportes o bien, brotes de lianas de mayor diámetro cuyos tallos se encuentran bajo tierra.

La distribución de los tallos de lianas en clases de tamaño fue más uniforme en los bosques nativos que en el bosque invadido. En estos últimos, los tallos de lianas de menor diámetro dominaron y casi no se encontraron tallos de lianas de mayor tamaño (e.g. $>4 \mathrm{~cm}$ de diámetro), de forma similar a lo que sucede con el diámetro de las lianas en los bosques clareados (Ding \& Zang 2009). Puede suceder que las lianas se encuentren en una etapa de establecimiento reciente en los bosques invadidos, por ello todavía no alcanzaron un diámetro importante, o bien, L. lucidum puede estar impidiendo su crecimiento, del mismo modo que disminuye la regeneración de otras especies nativas leñosas (Lichstein et al. 2004).

La diversidad baja de lianas en el bosque invadido tiene consecuencias en la conexión de estratos y en la dinámica del bosque. La falta de lianas disminuye la conexión de estratos en el bosque invadido y esto reviste importancia porque una de las funciones más importantes de las lianas es trepar árboles de diferente altura (Putz 1984) actuando como corredores de animales y dando continuidad al dosel (Emmons \& Gentry 1983). Una consecuencia de la presencia de lianas es el incremento en la mortalidad y la reducción en el crecimiento de los hospedadores, debido al sombreado, a su carga y derribando los árboles conectados cuando uno de ellos se cae (Putz 1984; Clark \& Clark 1990; van der Heijden et al. 2008). Entonces la falta de lianas en el bosque invadido puede contribuir probablemente de alguna forma a la persistencia de la especie invasora debido a que no se presentan lianas que regulen su mortalidad y crecimiento. 


\section{CONCLUSIONES}

Los bosques secundarios nativos y el bosque invadido de la sierra de San Javier presentaron diferente abundancia y composición florística de lianas. Los bosques nativos presentaron cuatro veces mayor abundancia y una mayor riqueza de tallos de lianas. El bosque invadido, dominado por la especie Ligustrum lucidum, presentó tallos de lianas en baja abundancia y asociados principalmente a las pocas especies de árboles nativos que se encuentran en este tipo de bosque. Casi ningún individuo de L. lucidum hospedó lianas y su dominancia se relacionó con una disminución en la abundancia de las plantas trepadoras. En el bosque invadido las lianas tuvieron una mayor proporción de individuos de menor diámetro, por lo cual es factible que su crecimiento se encuentre influenciado por la especie invasora y probablemente está influencia se mantenga en el tiempo debido a que L. lucidum tiene capacidad de auto-remplazo. En conclusión, L. lucidum afecta a las comunidades de lianas directa e indirectamente. La influencia directa radica en que los árboles de L. lucidum no constituyen soportes adecuados para las lianas y la influencia indirecta se manifiesta a través de la exclusión de árboles nativos hospedadores de lianas.

Agradecimientos: A R Fernández, S Rotger, G Bravo, G Sacchetti y H Ceballos que colaboraron con el trabajo de campo. A M Ayup por la ayuda logística. Al Instituto de Ecología Regional de la Universidad Nacional de Tucumán por el préstamo del material para los muestreos y por permitir el uso de su base de datos de parcelas permanentes. A dos revisores anónimos que contribuyeron con sus comentarios a mejorar el manuscrito.

\section{BiBLIOGRAFÍA}

Addo-Fordjour, P; S Obeng; MG Addo \& S Akyeampong. 2009. Effects of human disturbances and plant invasión on liana community structure and relationship with trees in the Tinte Bepo forest reserve, Ghana. Forest Ecol. Manag., 258:728-734.

Aragón, R \& M Groom. 2003. Invasion by Ligustrum lucidum (Oleaceae) in NW Argentina: early stage characteristics in different hanitat types. Rev. Biol. Trop., 51:59-70.

Aragón, R; L Montti; MM Ayup \& R Fernández. 2014. Exotic species as modifiers of ecosystem processes: Litter decomposition in native and invaded secondary forests of NW Argentina. Acta Oecol., 54:21-28.

AyARDE, HR. 2005. Vegetación lianescente de las áreas montanas del noroeste de Argentina. Lilloa, 42(1-2): 95128.

AYuP, MM; L MONTTI; R ARAGÓN \& HR GRAu. 2014. Invasion of Ligustrum lucidum (Oleaceae) in the southern Yungas: Changes in hábitat properties and decline in bird diversity. Acta Oecol., 54:72-81.

BIANCHI, AR. 1981. Las precipitaciones del Noroeste
Argentino. Instituto Nacional de Tecnología Agropecuaria. Salta, Argentina. 388 pp.

Brown, AD; A Grau; T LOMÁscolo \& NI Gasparri. 2002. Una estrategia de conservación para las selvas subtropicales de montaña (Yungas) de Argentina. Ecotrópicos, 15: 147-159.

Caballé, G \& A Martin. 2001. Thirteen years of change in trees and lianas in a Gabonese rainforest. Plant Ecol., 152:167-173.

Cabrera, AL. 1971. Fitogeografía de la República Argentina. Bol. Soc. Argent. Bot., 14:1-42.

Campanello, PI; JF Garibaldi; MG Gatti \& G Goldstein. 2007. Lianas in a subtropical Atlantic Forest: Host preference and tree growth. Forest Ecol. Manag., 242: 250-259.

Clark, DB \& DA Clark. 1990. Distribution and effects on tree growth of lianas and woody hemepiphytes in a Costa Rican tropical wet forest. J. Trop. Ecol., 6: 321-331.

Dewalt, SJ; SA Schnitzer \& JS Denslow. 2000. Density and diversity of lianas along a chronosequence in a central Panamanian lowland forest. J. Trop. Ecol., 16:1-19.

DING, Y \& R ZANG. 2009. Effects of logging on the diversity of lianas in a lowland tropical rain forest in Hainan Island, South China. Biotropica, 41: 618-624.

Easdale, TA; JR Healey; HR Grau \& A Malizia. 2007. Tree life histories in a montane subtropical forest: species differ independently by shade-tolerance, turnover rate and substrate preference. J. Ecol., 95:1234-1249.

EMmONs, LH \& AH GENTRY. 1983. Tropical forest structure and the distribution of gliding and prehensive-tailed vertebrates. Am. Nat., 121:513-524.

Furey, C; PA Tecco; N Perez-Harguindeguy; MA Giorgis \& M Grossi. 2014. The importance of native and exotic plant identity and dominance in descomposition patterns in mountain woodlands of central Argentina. Acta Oecol., 54: 13-20.

Gavier-Pizarro, GI; T Kuemmerle; LE Hoyos; SI Stewart; CD HUEBNER ET AL. 2012. Monitoring the invasion of an exotic tree (Ligustrum lucidum) from 1983 to 2006 with Landsat TM/ETM + satellite data and Support Vector Machines in Córdoba, Argentina. Remote Sens. Environ., 122: $134-145$.

Gelman, A \& J Hill. 2007. Data Analysis Using Regression and Multilevel/Hierarchical Models. Analytical methods for social research. Cambridge University Press. 625 pp.

Gentry, AH. 1991. Distribution and evolution of climbing plants. In: Putz F.E. \& H.A. Mooney (Eds.). The Biology of Vines. Pp. 3-49. Cambridge University Press, Cambridge, UK.

Gerwing, JJ; SA Schnitzer; RJ Burnham; F Bongers; J ChAVE ET AL. 2006. A standard protocol for liana censuses. Biotropica, 38: 56-261.

Giorgis, MA; AM Cingolani; F Chiarini; J Chiapella; G BARBOZA ET AL. 2011a. Composición florística del Bosque Chaqueño Serrano en la provincia de Córdoba, Argentina. Kurtziana, 36(1): 9-43.

Giorgis, MA; PA Tecco; AM Cingolani; D Renison; P MARCORA \& V PAIARO. 2011b. Factors associated with woody alien species distribution in a newly invaded mountain system of central Argentina. Biol. Inv., 13: 1423-1434.

Grau, HR; MF Arturi; AD Brown \& PG Aceñolaza. 1997. Floristic and structural patterns along a chronosequence of secondary forest succession in Argentinean subtropical 
montane forest. Forest Ecol. Manag., 95:161-171.

GrAU, HR \& R ARAgÓN. 2000. Árboles invasores de la Sierra de San Javier. En: Grau H.R. \& R. Aragón (Eds.). Árboles exóticos de las Yungas Argentinas. Pp. 5-20. LIEY-UNT, Tucumán, Argentina.

Grau, HR; ME Hernández; J Gutierrez; NI Gasparri; MC CASAVECCHIA ET AL. 2008. A peri-urban neotropical forest transition and its consequences for environmental services. Ecol. Soc., 13:35.

Grau, HR; L Paolini; A Malizia \& J Carilla. 2010. Distribución, estructura y dinámica de los bosques de la sierra de San Javier (Tucumán, Argentina). En: Grau H.R. (Ed.). Ecología de una interfase natural urbana, La sierra de San Javier y el Gran San Miguel de Tucumán. Pp. 33-50. Edunt. Tucumán, Argentina.

Hegarty, Ee \& G Caballé. 1991. Distribution and abundance of vines in forest communities. In: Putz, FE \& HA Mooney (Eds.). The Biology of Vines. Pp. 313-335. Cambridge University Press, Cambridge, UK.

Hoyos, LE; GI Gavier-Pizarro; T Kuemmerle; EH Bucher; VC RADELOFF \& PA TECCO. 2010. Invasion of glossy privet (Ligustrum lucidum) and native forest loss in the Sierras Chicas of Córdoba Argentina. Biol. Inv., 12:3261-3275.

Huston, M. 1995. Biological diversity: the coexistence of species in changing landscapes. 1st ed. Cambridge University Press, Cambridge, UK.

LichSteIN, JW; GA RicARDO \& R ARAGÓn. 2004. Recruitment limitation in secondary forests dominated by an exotic tree. J. Veg. Sci., 15:721-728.

LONDRÉ, RA \& SA SCHNITZER. 2006. The distribution of lianas and their change in abundance in températe forest over the past 45 years. Ecology, 87:2973-2978.

MALIZIA, A. 2003. Host tree preference of vascular epiphytes and climbers in a subtropical montane cloud forest of Northwest Argentina. Selbyana, 24(2): 196-205.

Malizia, A \& HR GRAu. 2006. Liana-host tree associations in a subtropical montane forest of north-western Argentina. J. Trop. Ecol., 22: 331-339.

Malizia, A \& HR Grau 2008. Landscape context and microenvironment influences on liana communities within treefall gaps. J. Veg. Sci., 19: 597-604.

Malizia, A; HR Ayarde \& Y Sasal. 2009. Ecología y diversidad de lianas en la selva pedemontana de las Yungas Australes. En: Brown, AD; PG Blendinger; T Lomáscolo \& P García Bes. Selva Pedemontana de las Yungas, Historia natural, ecología y manejo de un ecosistema en peligro. Pp. 75-104.

Malizia, A;HR Grau \&JW Lichstein. 2010. Soil phosphorus and disturbance influence liana communities in a subtropical montane forest. J. Veg. Sci., 21:551-560.

Mccune, B \& JB Grace. 2002. Analysis of Ecological Communities. MjM Software Design. Gleneden Beach, US.

Mccune, B \& MJ MefFord. 2006. PC-ORD 5.0. Multivariate analysis of ecological data. Mjm Software Design, Gleneden Beach, US.

Montaldo, NH. 2000. Éxito reproductivo de plantas ornitócoras en un relicto de selva subtropical en Argentina. Rev. Chil. Hist. Nat., 73: 511-524.

Pérez-Salicrup, DR \& W DE Meijere. 2005. Number of lianas per tree and number $f$ trees climbed by lianas at Los Tuxtlas, Mexico. Biotropica, 37:153-156.

Putz, FE. 1984. The natural history of lianas on Barro Colorado Island, Panamá. Ecology, 65:1713-1724.

PuTz, FE. 1990. Liana stem diameter growth and mortality rates on Barro Colorado Island, Panamá. Biotropica, 22(1): 103-105.

Putz, FE \& NM HoLвRоок. 1991. Biomechanical studies of vines. En: Putz F.E. y H.A. Mooney (Eds.). The Biology of Vines, pp. 73-97. Cambridge University Press, Cambridge, UK.

R Core Team. 2013. R: A language and environment for statistical computing. R Foundation for Statistical Computing, Vienna, Austria. URL http://www.Rproject.org/

SCHNITZER, SA \& F BONGERs. 2002. The ecology of lianas and their role in forests. Trends. Ecol. Evol., 17:223-229.

Van Der Heidden, GMF; JR Healey \& OL Phillips. 2008. Infestation of trees by lianas in a tropical forest in Amazonian Peru. J. Veg. Sci., 19:747-756.

Zamora Nasca, L; L Montti; R Grau \& L PaOlini. 2014. Efectos de la invasión del ligustro, Ligustrum lucidum, en la dinámica hídrica de las Yungas del noroeste argentino. Bosque, 35): 195-205. 